\title{
Redundant Dissimilar Sensor Fusion with Dynamic Driver Input Classification and Graceful Degradation for Drive-by-Wire Applications
}

\author{
Neal Y. Lii \\ Institute of Robotics and Mechatronics \\ German Aerospace Center \\ Oberpfaffenhofen, Germany \\ neal.lii@dlr.de
}

\author{
Stefan Sturm \\ The BMW Group \\ Munich, Germany \\ stefan.sa.sturm@bmw.de
}

\author{
Timothy A. Coombs \\ Department of Engineering \\ University of Cambridge \\ Cambridge, United Kingdom \\ tac1000@cam.ac.uk
}

\begin{abstract}
Dissimilar sensor redundancy with force and displacement measurements can provide extended fault coverage for drive-by-wire applications. However, large variances occur when correlating these inputs in dynamic measurements. To address this issue, this paper introduces a novel fault tolerant sensor fusion concept to fuse redundant kinetic and kinematic sensors dynamically and accurately. It consists of a driver input condition classifier, and a graceful degradation scheme voter. The classifier determines the states of several driver input conditions in real-time, which are then utilized as a pointers to allocate the best fitting driver input kinetic-kinematic response profile during dynamic operation for sensor data conversion. The graceful degradation voter module facilitates voting and fusing of the converted redundant sensor data. Functional safety is further maintained through a graceful degradation scheme to tolerate the presence of different fault scenarios in the system. The proposed sensor fusion concept is implemented on a brake-by-wire pedal test bench. Test results show significant performance gains in driver command accuracy for fault-tolerant dissimilar redundant sensor fusion.
\end{abstract}

Keywords- fault tolerance; drive-by-wire; driver input; fuzzy system; dissimilar sensor fusion; redundancy; voter algorithms

\section{INTRODUCTION}

\section{A. Drive-by-Wire Driver Input Interface Concepts}

The driver input interface is an integral part of a drive-bywire system. Instead of conventional mechanical linkages, the driver command can be produced utilizing a variety of sensors measuring different properties of the driver input. Several driver input interface formats have been proposed in the past to simulate conventional mechanical driver interfaces for to help gain public acceptance, such as the pedal cluster, and the steering wheel. New driver input formats, such as the joystick, which explores new driver input design freedom, have also been implemented in test vehicles.

Due to the safety-critical nature of drive-by-wire, these driver input interface designs must employ redundancy concepts to ensure sufficient fault tolerance. In order to extend fault coverage, dissimilar redundancy concepts should be considered. Several by-wire pedal cluster concepts have been proposed with redundant sensors measuring different input properties [1][2][3]. Examinations of different similar and dissimilar redundancy schemes have shown that through the utilization of sensors measuring decoupled driver input information (e.g. force and displacement), fault coverage can be further extended [4] [5].

\section{B. Redundant Sensor Fusion Algorithms}

Several sensor fusion algorithm approaches have been proposed in previous studies, which may be employed to fuse redundant sensor inputs. A common theme is the integration of smart algorithms into the fault-tolerance voter [6].

One smart voting algorithm is the smoothing voter [7]. This concept may be viewed as an extension of the threshold voter by adding a dynamic adaptive function to the threshold specification. This concept provides a compromise between accuracy and availability. If the majority voter could not produce a consensus outcome with the current threshold, the adaptive threshold specification would be widened until a voter outcome can be produced.

Another strategy is the fuzzy rule-based soft voter [8]. In this concept, different levels of faultiness are specified in the rule-based algorithm. Depending on the determined level of faultiness of a sensor channel, the weighting each sensor is adjusted accordingly to reduce the influence of the faulty channel.

The adaptive majority voter keeps a historical record to help determine the reliability of each sensor channel [9]. In this concept, a rolling historical record is kept for each sensor channel over a period of time. The resulting fused output is a majority consensus based on the reliability of each redundant channel to.

Several forms of adaptive voting have been applied in pervious work to a brake-by-wire driver input concept employing force and displacement sensors [1]. However, in this driver input sensor fusion implementation, driver input appears to be considered in only in static measurement. Driver input tests carried out in this work have shown that kinetic (e.g.

The work presented in this paper has been a part of a fault tolerant driveby-wire sensor fusion research partially sponsored by The BMW Group. It has been conducted at BMW AG, Munich, Germany, and Cambridge University, UK. 
force) and kinematic (e.g. displacement) measurements can experience large correlation variances during dynamic measurement. This can impair voter performance, resulting in increased voter error, and/or reduced fused output precision.

\section{Overview of this Paper}

This paper presents a new sensor fusion concept to address the problem of kinetic-kinematic sensor correlation variance in dynamic measurement. Section II describes the chosen kinetickinematic hardware sensor redundancy concept [5], as well as its implementation into a brake-by-wire pedal test bench. The proposed novel sensor fusion algorithm is described in Section III. It employs a dynamic driver input condition classifier to help allocate the best fitting kinetic-kinematic input correlation profile for the conversion of dissimilar sensor inputs for voting. The fusion algorithm continues with a threshold voter employing a graceful degradation scheme, which fuses the converted inputs to form the driver command output. The graceful degradation scheme allows an accurate driver command to be delivered even as the system experiences a variety of faults. Section IV presents the results and findings from this work. Finally, Section $\mathrm{V}$ provides a conclusion to this paper.

\section{The DuAl DeCOUPLED PAIR REDUNDANCY CONCEPT AND IMPLEMENTATION}

The Dual Decoupled Pair Redundancy Concept (DDP) introduces several strategies for implementing kinetic and kinematic measurements to achieve a wide range of fault coverage to help achieve improved fault-tolerance over previous by-wire redundancy fault tolerance concepts [5]. The main concepts of DDP are listed below, and illustrated in Fig. 1 for further clarification.

- Independent redundant power sources to ensure dependable power supply in case of a power source fault

- Utilization of sensors measuring decoupled driver input information (e.g. force and displacement), with a minimum of two of each sensor types to facilitate a variety of fault recognition and containment

- Sufficient physical separation between sensor clusters to help isolate and quarantine faults

- Direct tactile contact with the driver to eliminate potential introduction of driver input measurement faults

A catalogue of potential faults, which may be experienced by a driver input device, has been collected for this work. These faults have been classified into the following categories: data processing unit faults, software faults, sensor faults, data transmission faults, electrical connection faults, power supply faults, and mechanical faults. A failure mode and effect analysis (FMEA) of the DDP redundancy implemented for brake-by-wire has demonstrated the ability to provide coverage for 43 fault scenarios in these fault categories.

The DDP concept has been realized in the form of a pedal cluster incorporating two resistive thin-film force sensors on the pedal surface for measurement of the driver input force normal to the pedal, and two hall sensors for angular displacement measurements at the pedal rotational linkage (Fig. 2 left). A programmable hydraulic actuator (Fig. 2, right) provides repeatable driver input simulation for different conditions.

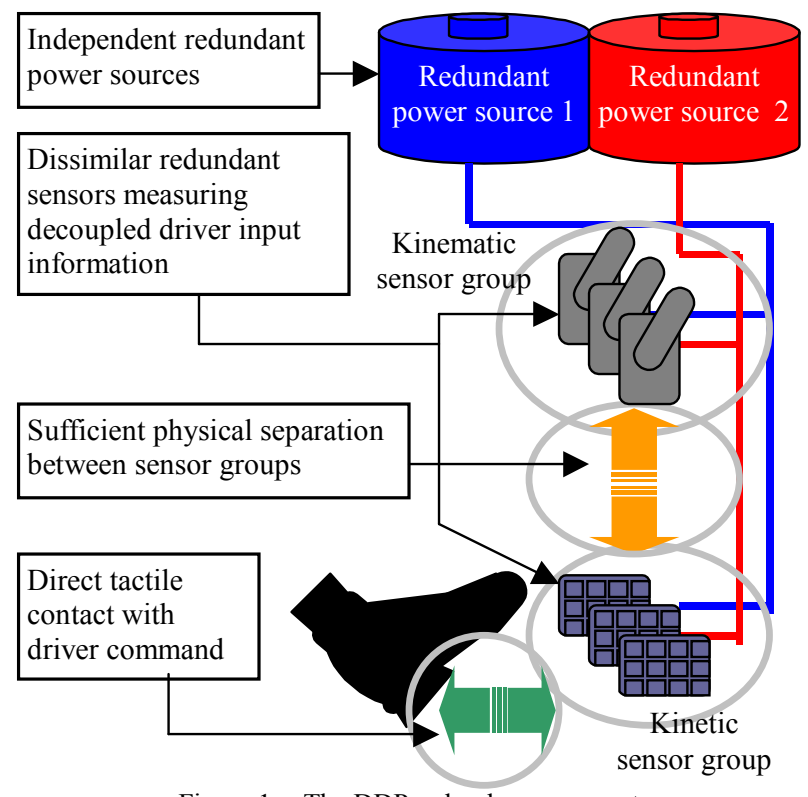

Figure 1. The DDP redundancy concept

\section{SENSOR FUSION ALGORITHM}

To enable DDP dissimilar sensor fusion with kinetic and kinematic driver inputs, a correlation must be drawn between the dissimilar inputs to commence voting. A kinetic-kinematic measurement relationship profile provides the correlation between the dissimilar sensors. Fig. 3 illustrates some examples of correlation profiles in different dynamic pedal operation. It can be clearly seen, that profile separations occur under different dynamic pedal input conditions. These kinetickinematic profile separations can yield large variances. Measurements taken in this work have shown variances as high as $29.4 \%$. Such variances would significantly hinder the fused output precision. This would be unacceptable for brake or steering functions. The proposed algorithm aims to improve the precision of the fused sensor output in dynamic conditions.
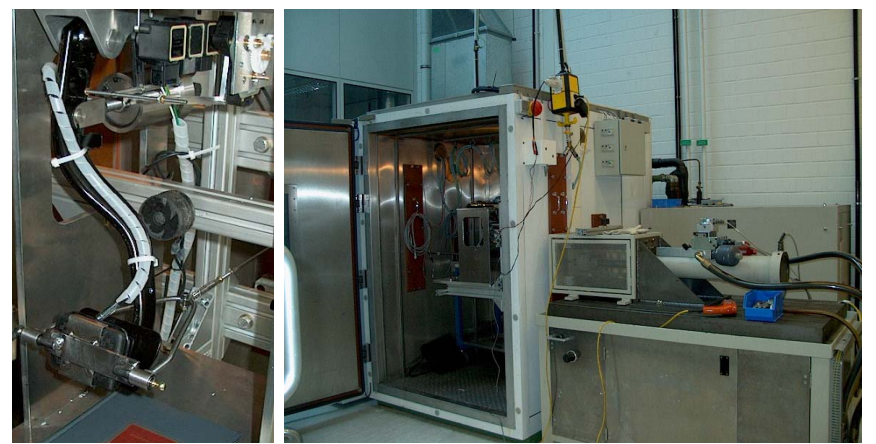

Figure 2. Brake-by-wire pedal test bench and experimental setup

A. Dynamic Driver Input Classification 
First, the measurement variance causes must be identified. Three driver input conditions have been identified in this work to influence kinetic-kinematic input correlation: drive input direction, distance, and speed, as shown in Fig. 3.

(a)

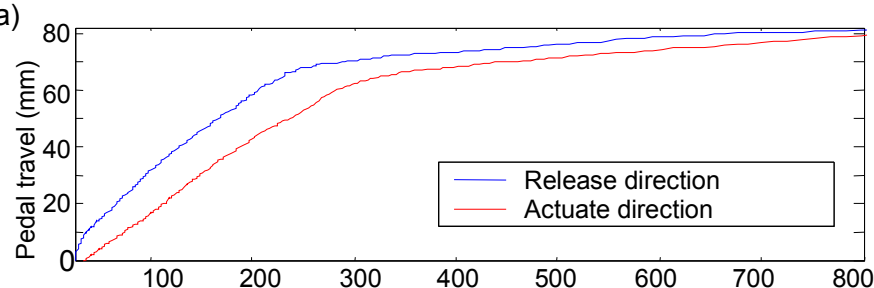

(b)

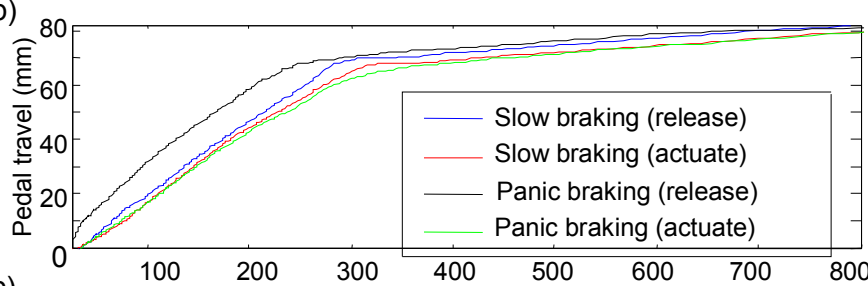

(c)

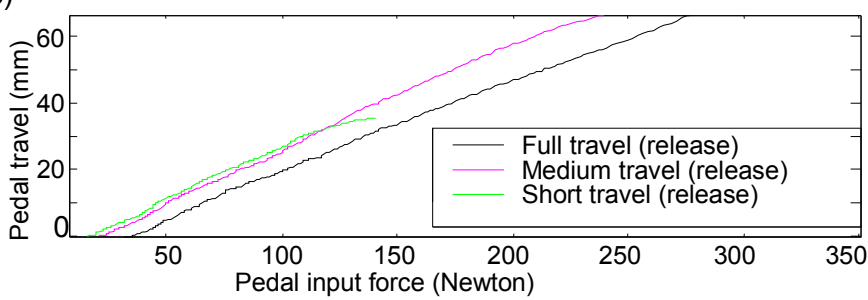

Figure 3. Examples of kinetic-kinematic sensor input correlation profile separation from dynamic measurements: (a) input direction, (b) input speed and (c) input distance

Previous fault tolerant sensor fusion concepts process dissimilar sensor inputs directly through smart algorithms to cope with redundant channel variance. The sensor fusion scheme proposed here introduces the addition of a conversion module to the voter design, as shown in Fig. 4. By separating out the dynamic variance processing from the voting task, the voter module design can be simplified, which helps reduce processing power requirements. Furthermore, the voter design can then be focused on fault recognition, with narrower fault trigger thresholds to improve fault containment performance.

The proposed conversion module, shown in more detail in Fig. 5, consists of two components: the driver input condition classifier, and the kinetic-kinematic input converter. The driver input condition classifier uses various features extracted from the raw sensor measurement of the driver input to deduce the current state of different driver input condition properties of speed, direction, and input distance.

The kinetic-kinematic relationship of the pedal unit can be described as a mass-spring-damper system, as shown below:

$$
f=k x+c x
$$

This relationship gives a correlation between force $f$, displacement $x$, and velocity $d x / d t$ of the pedal input. As a result, the pedal input force may be described as functions, $g$, of displacement and velocity, as shown in eq. (2):

$$
f=g_{1}(x)+g_{2}(x)
$$

In order to utilize the three kinetic-kinematic correlation varying input condition properties as identified in this work, this expression can be expanded into the following:

$$
f=g_{\text {speed }}+g_{\text {direction }}+g_{\text {distance }}
$$

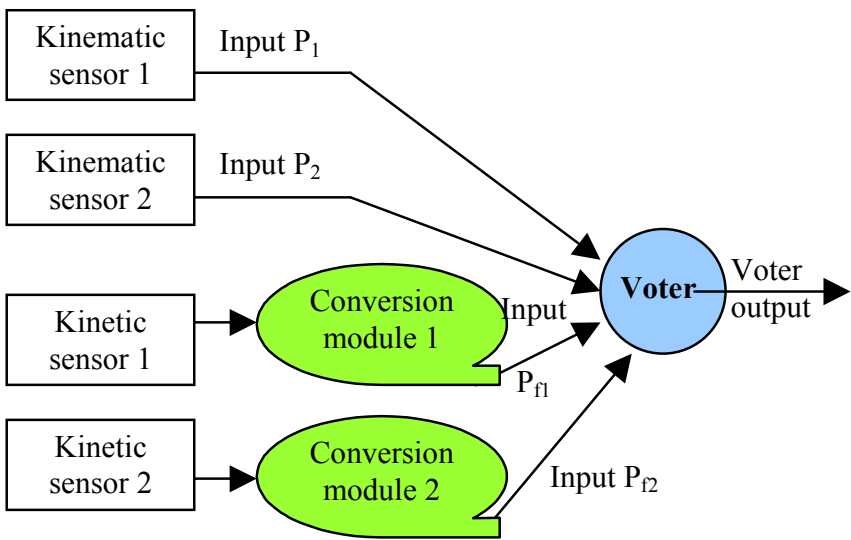

Figure 4. The sensor fusion voter proposed in this work, with conversion modules for dissimilar sensor input conversion

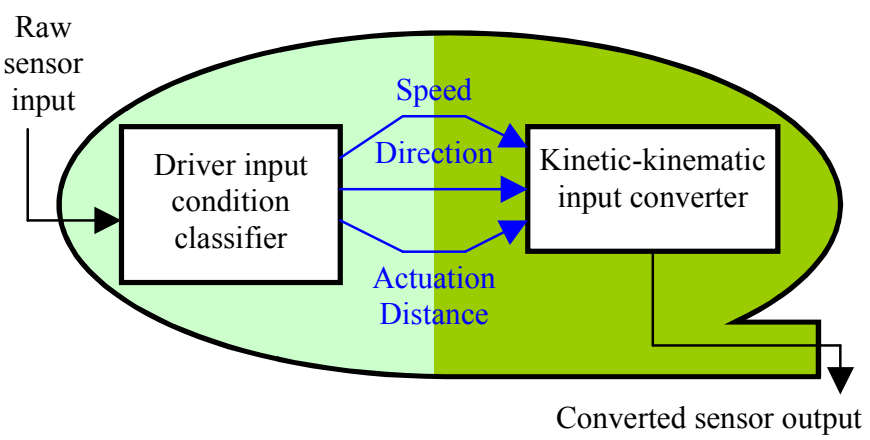

Figure 5. The dynamic sensor conversion module

The dynamic classification by the driver input condition classifier is achieved with fuzzy logic based driver input condition recognition [10]. Two classifiers are implemented for each sensor channel: a speed and direction classifier, and a pedal travel classifier. The module processes sensor inputs at 10 msec cycles in real-time, with the aid of a historical stack of up to $80 \mathrm{msec}$ to provide sufficient information for classification.

TABLE I. Possible Driver INPUT Classification TyPes of THE DRIVER INPUT CONDITION CLASSIFIER

\begin{tabular}{|c|l|l|l|}
\hline \multirow{2}{*}{} & \multicolumn{2}{|c|}{ Speed and Direction Classifier } & \multicolumn{1}{|c|}{$\begin{array}{c}\text { Pedal Travel } \\
\text { Classifier }\end{array}$} \\
\cline { 2 - 4 } & \multicolumn{1}{|c|}{ Speed } & \multicolumn{1}{|c|}{ Direction } & \multicolumn{1}{c|}{ Input Distance } \\
\hline \multirow{4}{*}{$\begin{array}{c}\text { Driver input } \\
\text { classification } \\
\text { types }\end{array}$} & $\begin{array}{l}\text { Static pedal } \\
\text { input }\end{array}$ & $\begin{array}{l}\text { Pedal } \\
\text { actuation }\end{array}$ & Short pedal travel \\
\cline { 2 - 4 } & $\begin{array}{l}\text { Light pedal } \\
\text { input }\end{array}$ & Pedal release & $\begin{array}{l}\text { Medium pedal } \\
\text { input }\end{array}$ \\
\cline { 2 - 4 } & Fast pedal input
\end{tabular}


In order to utilize the long historical stack data at a refresh rate acceptable for automotive brake command function, the $\mathrm{N}$-Tuple sliding frame technique has been employed to provide sensor data at $10 \mathrm{msec}$ cycles [11]. The drive-input classification is performed at $20 \mathrm{msec}$ cycles. To avoid fault contamination into other channels, each classifier is dedicated to an assigned sensor, and considers only raw and processed input data from this channel. The speed and direction classifier consists of 52 fuzzy inference rules, to recognize five speed, and two direction types, whereas the input distance classifier consists of 12 rules, classifying four pedal input travel ranges. These classifications are detailed in Table I.

\section{B. Kinetic-Kinematic Sensor Data Conversion}

With the driver input condition determined, an accurate kinetic-kinematic relationship profile can be established for the sensor date conversion. In this work, the kinetic sensor channels are converted with the aid of a lookup function $T$ to take the three driver input conditions as pointers to locate the best fitting correlation profile, as shown in eq. (4):

$$
P F_{i}(t)=T \text { (speed, direction, travel distance) }
$$

In order to ensure the availability of sensor channel conversion output, situations of multiple classification (e.g. recognition as both "medium" and "long" pedal travel), as well as missed classification must be resolved. In occurrences of multiple classifications to a sensor channel input, an average would be taken from the conversions based on all the classified driver input conditions taken from conversions based on each corresponding kinetic-kinematic profile, as shown in eq. (5). ClassifierOut(t) is a boolean term signifying the recognition of at the driver input time $(t)$ a certain driver input state, with 1 signifying recognition, and 0 for nonrecognition. $P F_{i}$ denotes the converted sensor channel data:

$$
P F_{\text {output }}=\frac{\sum_{i=1}^{n} \text { ClassifierOut }_{i}(t) \bullet P F_{i}(t)}{\sum_{i=1}^{n} \text { ClassifierOut }_{i}(t)}
$$

In occurrences of missed classification, the classifier output of the previous data delivery cycle would be utilized to facilitate sensor conversion, as expressed in eq. (6).

$$
P F_{\text {output }}=\frac{\sum_{i=1}^{n} \text { ClassifierOut }_{i}(t-1) \bullet P F_{i}(t)}{\sum_{i=1}^{n} \text { ClassifierOut }_{i}(t-1)}
$$

\section{Graceful Degradation Threshold Voter}

A threshold voter is implemented in the proposed graceful degradation scheme. Due to the high precision performance of the fuzzy classifier based sensor conversion, the threshold of the proposed voter may be reduced to a narrower range, which results in higher driver command precision. The graceful degradation voter scheme is shown in Fig. 6 to better illustrate its coverage of different fault scenarios. In the DDP concept, the sensor channels are grouped such that in case of one power source failure, a dissimilar sensor pair remains in correct functioning state. The power source fault check forms the first stage of the graceful degradation scheme. This is followed by a threshold check on whether any fault is present. Under normal condition, all sensors channels are fused as an average to form a driver command output. In the following stage of the graceful degradation, fault(s) are determined to be present, where an absolute majority threshold vote is commenced to eliminate a single faulty channel. If no absolute majority is obtained, mechanical fault recognition is attempted in the following stage. This is accomplished by obtaining sensor data agreement between similar sensors, but disagreement between dissimilar sensors. Finally, when no mechanical fault is determined to be present, a plurality (simple majority) vote is carried out to obtain a fused output.

\section{RESULTS AND DISCUSSION}

Experiments have been conducted on the test bench to provide data sets with over 50000 samples for each sensor channels collected at $10 \mathrm{msec}$ cycles. The proposed conversion module with dynamic classification yielded a peak variance of $3.94 \%$, which represents a large improvement over the $29.4 \%$ observed on conversion without dynamic driver input classification. Fig. 7 shows a conversion performance comparison. Fig. 8 shows the results of the converted kinetic sensor data as compared to the kinematic data. A close match can be clearly seen between the converted kinetic data and actual kinematic measurement.

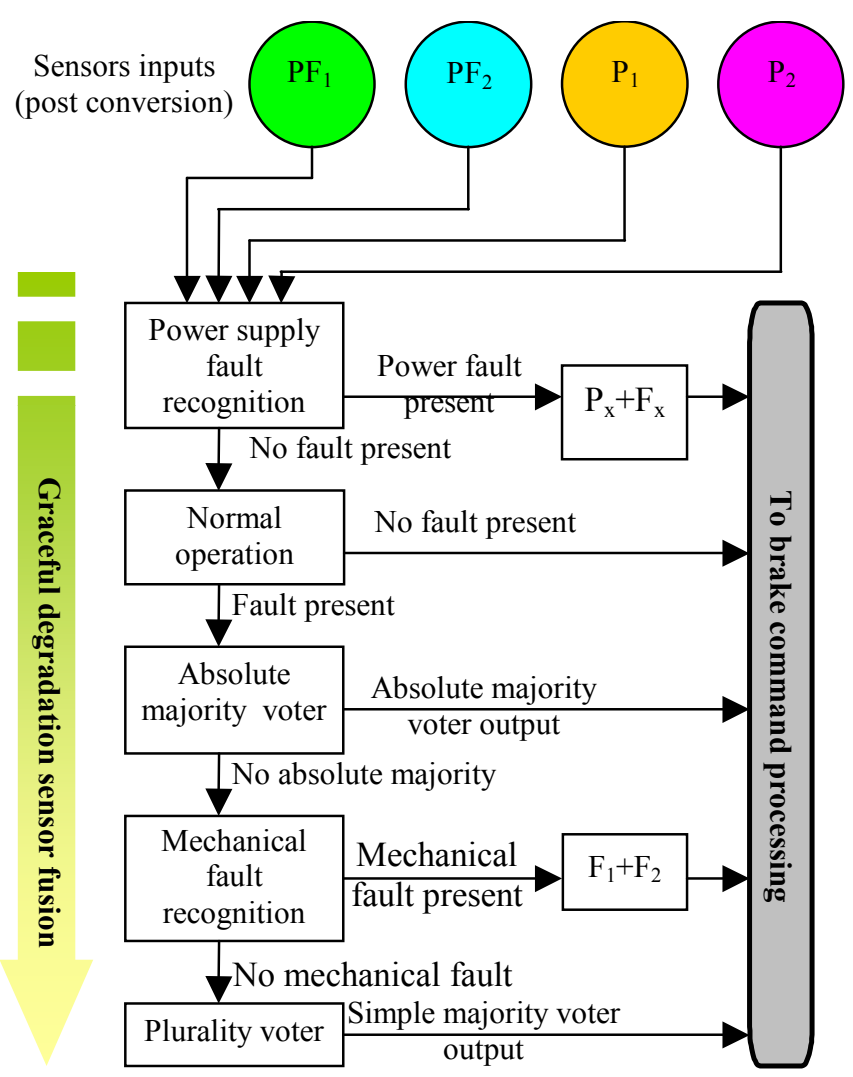

Figure 6. The proposed graceful degradation scheme facilitated with threshold voting 


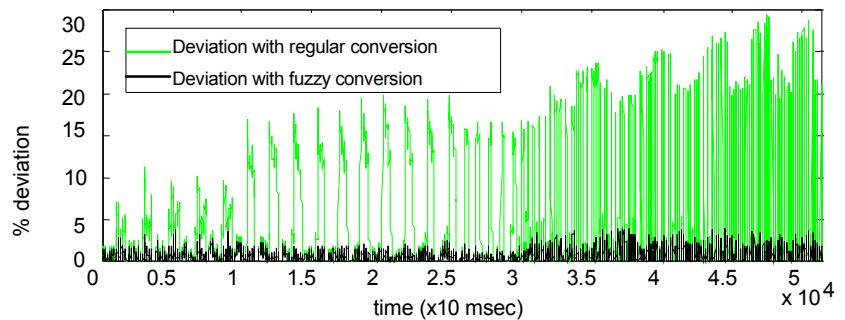

Figure 7. Improvement of the sensor variance with the proposed driver input condition classifier conversion versus regular conversion
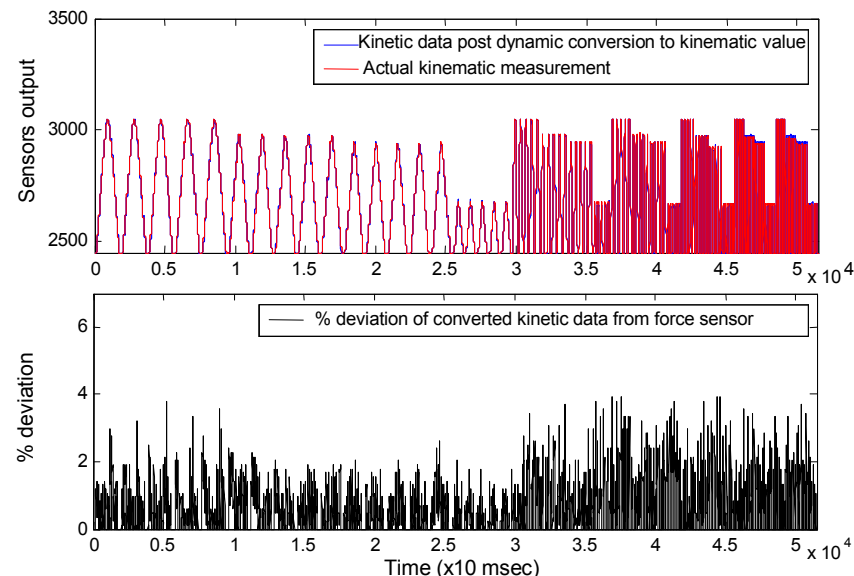

Figure 8. Results of dynamic conversion of kinetic sensors input to kinematic values

In order to examine the performance of proposed voter with graceful degradation scheme, experiments were carried out, simulating 43 fault scenarios in the six fault categories described in Section II. For different fault scenarios, faults have been injected at random to help examine the proposed system's ability to deliver accurate driver intended command. Fig. 9 gives an example of the fused output from the graceful degradation scheme. The ability of the DDP concept to contain mechanical faults can be clearly seen, as the fused output (in blue) separates from the faulty kinematic input to reflect the driver's actual intended command. Overall, the proposed sensor fusion algorithm demonstrated precision levels with a peak variance of $3.51 \%$ from driver intended command. Table II gives a more detailed view of the proposed voter's performance against different fault categories.

TABLE II. VOTER PERFormance Under DifFerent Fault CONDITIONS

\begin{tabular}{|l|l|l|}
\hline $\begin{array}{l}\text { Pedal Unit Operation Fault } \\
\text { Categories }\end{array}$ & Peak Variance & Mean Variance \\
\hline Normal Operation & $2.14 \%$ & $0.36 \%$ \\
\hline Sensor Fault & $3.24 \%$ & $0.67 \%$ \\
\hline Mechanical Fault & $3.51 \%$ & $1.21 \%$ \\
\hline $\begin{array}{l}\text { Data communication and } \\
\text { connection fault }\end{array}$ & $2.56 \%$ & $0.43 \%$ \\
\hline Power source fault & $2.51 \%$ & $0.42 \%$ \\
\hline Common Mode failure & $3.51 \%$ & $0.82 \%$ \\
\hline
\end{tabular}

\section{CONCLUSION}

This paper presents a new sensor fusion concept to address the problem of dissimilar sensor input correlation variances in dynamic operating conditions. As these variances can be large, a voter may erroneously treat normal sensor data as faulty.
This paper introduces a sensor fusion voter concept with the addition of a conversion module with dynamic driver input condition classification to facilitate the allocation of the best fitting correlation profile. A threshold voter with a graceful degradation scheme completes the fusing of dissimilar sensors. Experimental results show significant improvement over systems without the proposed dynamic driver input condition classification and graceful degradation threshold voter scheme.
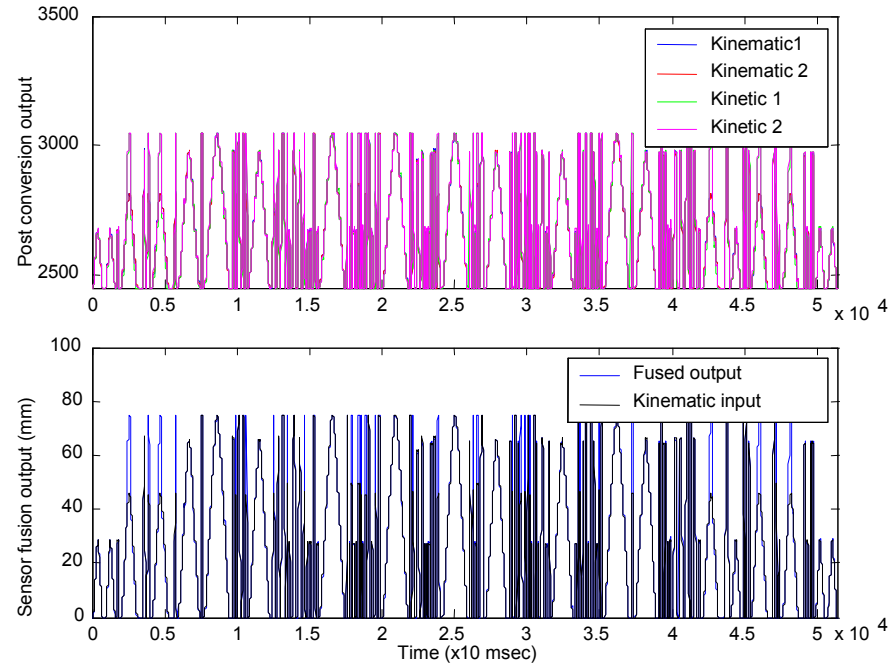

Figure 9. Example of the fused sensor output with the proposed dynamic conversion and graceful degradation scheme (mechanical jam failure case)

\section{REFERENCES}

[1] S. Stölzl, "Fault Tolerant Pedal Unit for an Electromechanical Brake System (Brake-by-Wire) / Fehlertolerante Pedaleinheit für ein elektronmechanisches Bremssystem (Brake-by-Wire)," $\mathrm{PhD}$ Dissertation, Institute of Automatic Control, Technical University of Darmstadt, 2000

[2] K. Slanec, "Safe and reliable - or just complex?" AutoTechnology, June 2002

[3] R. Hoseinnezhad, A. Bab-Hadiashar, and P. Harding, "Fusion of brake pedal sensors in by-wire cars: a fuzzy logic approach," Proceedings of the Third IFAC Symposium on Mechatronic Systems, Sydney, Australia, Volume 2, pp. 639-644, September 6-8, 2004

[4] N. Y. Lii, S. Sturm, and T. A. Coombs, "Driver input sensor selection and topologies for fault-tolerant drive-by-wire applications," Proceedings of the IEEE/ASME Conference on Advanced Intelligent Systems, Monterey, California, USA, pp. 122-127, July 24-28, 2005

[5] N. Y. Lii, "Fault-tolerant multi-sensor fusion for drive-by-wire driver interface design" $\mathrm{PhD}$ Dissertation, University of Cambridge, Cambridge, United Kingdom, April 2008

[6] B. Parhami, "Voting algorithms," IEEE Transactions on Reliability, Volume 43 (4) pp. 617-629, December, 1994

[7] G. Latif-Shabgahi, S. Bennett, and J.M. Bass, "Smoothing voter: a novel voting algorithm for handling multiple errors in fault-tolerant control systems," Microprocessors and Microsystems, Volume 27 (7): pp. 303-313, August 2003

[8] R. Hoseinnezhad, and A. Bab-Hadiashar, "Fusion of redundant information in brake-by-wire system using a fuzzy voter," Journal of Advanced Information Fusion, Volume 1 (1): pp. 52-62, July 2006

[9] G. Latif-Shabgahi, and S. Bennett, "Adaptive majority voter: a novel voting algorithm for real-time fault-tolerant control systems," Proceedings of the 25th Euromicro Conference, Volume 2, pp. 113-120, 8-10 September 1999

[10] W. Pedrycz, and F. Gomide, "An Introduction to Fuzzy Sets Analysis and Design", The MIT Press, Cambridge, MA, USA, 1998

[11] J. J. Yoon, "Single-Imager Occupant Detection Based on Surface Reconstruction," PhD Dissertation, City University, London, United Kingdom, October 2004 